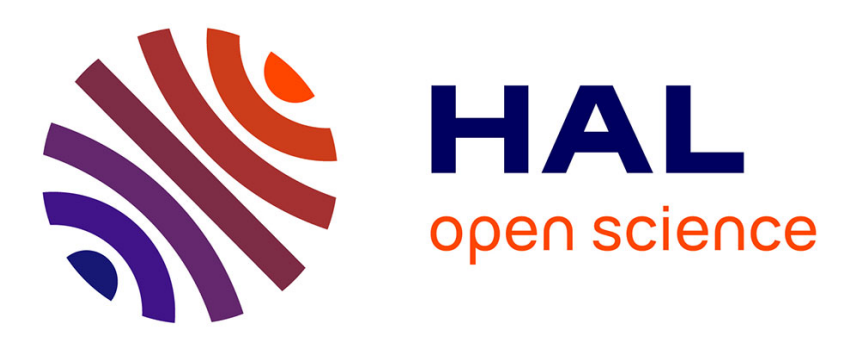

\title{
A substitution matrix for structural alphabet based on structural alignment of homologous proteins and its applications.
}

Manoj Tyagi, Venkataraman S. Gowri, Narayanaswamy Srinivasan, Alexandre de Brevern, Bernard Offmann

\section{To cite this version:}

Manoj Tyagi, Venkataraman S. Gowri, Narayanaswamy Srinivasan, Alexandre de Brevern, Bernard Offmann. A substitution matrix for structural alphabet based on structural alignment of homologous proteins and its applications.. Proteins - Structure, Function and Bioinformatics, 2006, 65 (1), pp.32-9. 10.1002/prot.21087 . inserm-00133760

\section{HAL Id: inserm-00133760 https://www.hal.inserm.fr/inserm-00133760}

Submitted on 4 Sep 2009

HAL is a multi-disciplinary open access archive for the deposit and dissemination of scientific research documents, whether they are published or not. The documents may come from teaching and research institutions in France or abroad, or from public or private research centers.
L'archive ouverte pluridisciplinaire HAL, est destinée au dépôt et à la diffusion de documents scientifiques de niveau recherche, publiés ou non, émanant des établissements d'enseignement et de recherche français ou étrangers, des laboratoires publics ou privés. 


\section{A substitution matrix for structural alphabet based on structural alignment of homologous proteins and its applications}

Manoj Tyagi ${ }^{1}$, Venkataraman S. Gowri ${ }^{2}$, Narayanaswamy Srinivasan ${ }^{1,2}$, Alexandre G. de Brevern ${ }^{3, *} \&$ Bernard Offmann ${ }^{1, *}$

${ }^{1}$ Laboratoire de Biochimie et Génétique Moléculaire, Université de La Réunion, BP 7151, 15 avenue René Cassin, 97715 Saint Denis Messag Cedex 09, La Réunion, France.

${ }^{2}$ Molecular Biophysics Unit, Indian Institute of Science, Bangalore 560 012, India.

${ }^{3}$ Equipe de Bioinformatique et Génomique Moléculaire, INSERM U726, Université Paris 7, case 7113, 2, place Jussieu, 75251 Paris Cedex 05, France.

*Corresponding author :

Bernard Offmann

Laboratoire de Biochimie et Génétique Moléculaire,

Université de La Réunion,

BP 7151, 15 avenue René Cassin,

97715 Saint Denis

Messag Cedex 09,

La Réunion, France.

Phone : +262 262938641

Fax : +262 262938237

bernard.offmann@univ-reunion.fr

Running title: Structural alphabet substitution matrix.

Number of pages: 24

Number of tables: 2

Number of figures: 4 


\section{Abstract}

Analysis of protein structures based on backbone structural patterns known as structural alphabets have been shown to be very useful. Among them, a set of 16 pentapeptide structural motifs known as protein blocks (PBs) has been identified and upon which backbone model of most protein structures can be built. Protein blocks allows simplification of 3D space onto 1D space in the form of sequence of PBs. Here, for the first time, substitution probabilities of PBs in a large number of aligned homologous protein structures has been studied and is expressed as a simplified $16 x 16$ substitution matrix. The matrix was validated by benchmarking how well it can align sequences of PBs rather like amino acid alignment to identify structurally equivalent regions in closely or distantly related proteins using dynamic programming approach. The alignment results obtained are very comparable to well established structure comparison methods like DALI and STAMP. Other interesting applications of the matrix have been investigated. We first show that, in variable regions between two superimposed homologous proteins, one can distinguish between local conformational differences and rigid-body displacement of a conserved motif by comparing the PBs and their substitution scores. Second, we demonstrate, with the example of aspartic proteinases, that $\mathrm{PBs}$ can be efficiently used to detect the lobe/domain flexibility in the multi-domain proteins. Lastly, using protein kinase as an example, we identify regions of conformational variations and rigid body movements in the enzyme as it is changed to the active state from an inactive state.

Keywords : Local protein structures, substitution matrix, structural alphabet, structure alignment and comparison, rigid body shift 


\section{Introduction}

It has been realized since long time that known protein structures can be re-generated by assembling fragments from a repertoire of short structural motifs. Many of these short structural motifs re-occur in a large number of proteins of diverse structure and function ${ }^{1-5}$. Analysis of protein structures based on these short structural motifs has been widely used by various groups and have been shown to be useful in protein structure prediction ${ }^{6-9}$, reconstruction of backbone ${ }^{10-13}$, description and prediction of small loops ${ }^{14-16}$ and long fragments ${ }^{17-19}$. Following these early leads, a set of 16 pentapeptide structural motifs have been identified ${ }^{20,21}$ as a set of basic backbone structural patterns known as "protein blocks" (hereafter referred as PBs). PBs represent basic structural motifs upon which backbone model of most protein structures can be built. They have been found to be very informative ${ }^{7,22}$ and useful for pre processing before $a b$ initio and new fold recognition. Interestingly, in a recent work ${ }^{23} \mathrm{PBs}$ has been used for protein $3 \mathrm{D}$ structure prediction Each $\mathrm{PB}$ is characterized by a set of $16(\phi, \psi)$ values and is represented by a character symbol $a$, $b, c, \ldots$ to $p$ (refer Materials and methods). A known protein structure can be encoded into PBs by sliding a overlapping window of five residues along the backbone and PBs for each window could be assigned on the basis of the smallest root mean square deviation on angular values ${ }^{24}$ between the observed $(\phi, \psi)$ values in the window and the standard torsion angle values for various PBs. Hence, 3D information of protein structure can be represented (simplified) into a 1D sequence of PBs.

It is now well documented that simplification of $3 \mathrm{D}$ space onto $1 \mathrm{D}$ space is an efficient tool to understand the sequence-structure relationship ${ }^{18,25}$ and opens up new front for structure analysis of proteins namely structure comparison and alignment. Reduction of 3D space onto 1D space using local structural properties, to align and 
identify structurally equivalent regions have been exploited earlier ${ }^{26}$. Flexible protein structure alignment and comparison methods, like SSAP $^{27,28}$ and DALI ${ }^{29}$ based on combination of distance matrices and dynamic programming technique have been present for long time and shown to be effective in both global ${ }^{28}$ and local ${ }^{30}$ structure alignment. Similarly, pairwise protein structure alignment using orientation independent backbone representation with dynamic programming has shown lot of promise $^{31}$. Analysis of protein structures in terms of sequences of structural alphabets (SAs) combined with alignment algorithm to find structural similarities have been reported recently in the form of a web service called SA-Search ${ }^{32}$.

Even though alignment of SAs using classical alignment algorithm has been tried out to some effective way, a genuine substitution matrix for SAs is required, similar to amino acid substitution matrix, to fully exploit the potential of such an approach. This requirement has been tentatively addressed in SA-Search where substitution scores were derived only from emission probabilities of hidden states in Hidden Markov Model ${ }^{32}$.This approach diverges from more classical methods.

Here we derive a substitution table for PBs on the basis of assignment of PBs to structurally aligned homologous proteins. These proteins are present in a large database, PALI ${ }^{33,34}$, of homologous protein families with structure-based alignment available for every family. The 16 x 16 PB substitution table provides extent of preference of a $\mathrm{PB}$ in a protein for its retention or substitution by any other $\mathrm{PB}$ in an aligned homologue. Usage of such methodology to extract substitution matrix provides a more rational approach over the matrix used in SA-Search, namely to evaluate equivalence between homologous structures. Among several possible uses of PB substitution table, we demonstrate, in this paper, its application to the following problems: 
* Aligning structures and identifying structurally equivalent regions between homologous or distantly related proteins using a dynamic programming approach.

* Distinguishing between conformational differences and rigid body shifts among homologous protein structures.

* Characterization of changes in structures between active and inactive states of enzymes, by taking protein kinases as an example.

\section{Materials and methods}

\section{Protein Blocks}

A structural alphabet that is able both to approximate 3D structure and to be useful in prediction process has been identified ${ }^{20}$. It is composed of 16 folding patterns of five consecutive residues, (PBs), representing local structural features of proteins. Description of how PBs were identified has already been documented ${ }^{20}$. Each of the PBs is represented by a vector of eight dihedral angles associated with five consecutive $\mathrm{C} \alpha$ atoms and the PBs are denoted by letters $a, b, \ldots, p$. These PBs represent distinct and most common backbone conformations of pentapeptide regions in proteins of known structure. As can be seen from Table 2, dihedral angle values for the PBs $d$ and $m$ correspond to the prototype for the central $\beta$-strand and the central $\alpha$ helix, respectively. PBs $a$ through $c$ primarily represent $\beta$-strand $\mathrm{N}$-caps and $e$ and $f$, C-caps. PBs $g$ through $j$ are specific to coils, $k$ and $l$ to $\alpha$-helix $\mathrm{N}$-caps, and $n$ through $p$ to $\alpha$-helix C-caps.

In order to assign PB to a pentapeptide region in a protein structure, root mean square deviations on angular values or $r m s d a^{24}$ between observed $(\phi, \psi)$ values in the pentapeptide and ideal $(\phi, \psi)$ values of each one of 16 PBs (Table 1) are calculated. 
The assigned PB to the pentapeptide region corresponds to the one with lowest rmsda. In this manner a $3 \mathrm{D}$ protein structure is translated into a $1 \mathrm{D}$ sequence of $\mathrm{PBs}$ representing structure information as sequence of structural alphabets.

\section{Database of aligned homologous protein structures}

The database of Phylogeny and Alignment of homologous protein structures (PALI $^{33,34}$ comprises of structure-based pairwise and multiple alignments of homologous proteins of known three-dimensional structure. The rigid-body superposition program $\mathrm{STAMP}^{35}$ has been used for this purpose. The database also consists of phylogenetic tree structures of various protein families derived using sequence-based and structure-based similarity measures. The PALI database is available at http://pauling.mbu.iisc.ernet.in/ pali.

All the structurally aligned homologous proteins from PALI database were translated into alignment of PB sequences. As one PB represents five $\mathrm{C} \alpha$ atoms, we have used a convention of associating the PB to the middle residue of the pentapeptide. Therefore for protein of length $N$, the length of PB sequence is $N-4$.

The dataset used in the current analysis consists of 1197 protein families with 6140 protein structures involved in 21503 pairwise alignments. The derivation of $16 \times 16$ PB substitution matrix is aided by 2071225 observations of PB substitutions in the homologous protein structures.

\section{Calculation of substitution matrix}

The number of substitutions between any two PBs is counted based only on the alignment corresponding to structurally conserved regions identified by STAMP superimposition. This caution is exercised, as the alignment of residues in the structurally variable regions is meaningless in the rigid body alignments. The raw 
frequencies are normalized with respect to the total number of two PBs in question as well as with respect to the total number of PB-PB substitutions in the dataset. These normalized frequencies are then expressed as the $\log$-odds scores as follows ${ }^{36}$ :

$$
\left.S_{i, j}=\log _{e} \mid \frac{N_{i j} / \sum_{j=1}^{16} N_{i j}}{\sum_{i=1}^{16} N_{i j} / \sum_{i=1}^{16} \sum_{j=1}^{16} N_{i j}}\right\rfloor
$$

where $N_{i j}$ is the raw frequency of replacing $\mathrm{PB} i$ with $\mathrm{PB} j$.

\section{Data set used for validation of PB matrix}

Validation of the substitution matrix was performed by using structural alignments to identify local equivalent regions. The initial goal was to benchmark our method (PBALIGN) using a comprehensive set of protein domain pairs. A total of 29 pairs was used in this evaluation, among which, 14 pairs were taken from Shindyalov and Bourne $^{37}$ and the other 15 pairs were taken randomly within SCOP families with a sequence identity cutoff of $40 \%$. The complete list is provided in supplementary data.

\section{Results}

The presented work is based on the concept of translating structurally aligned homologous proteins into aligned sequences of 16 types of PBs and calculation of PB substitution frequency to obtain a normalized 16x16 matrix This matrix gives a score to substitute a given PB into another in topologically equivalent regions.

\section{Substitution matrix}

Analysis of pairs of proteins from PALI database was used to construct a PB substitution matrix. Table 2 provides the final substitution matrix, which is used 
extensively in the work described in this manuscript. It can be noted that most of the off-diagonal elements are negative suggesting that conformations of most of the pentapeptides in the homologous protein structures are conserved. The following PBs pairs $c-a, f-e, g-a, g-c, g-e, h-e, i-a, j-b, j-h, j-i, k-h, n-g, o-h, p-b, p-g, p-i, p-j, b-h, b-i$, $b-l, c-d, j-k, k-l, l-o, n-o, o-p$ and $p-n$ are the off-diagonal elements with positive substitution scores i.e. favorable substitutions. Figure 1a and $1 \mathrm{~b}$ show examples of $g-a$ and $e-f$ substitutions respectively and these PB pairs show similarity in their structures in the middle of the segments. Figure 1c gives an example of negative score substitution indicating differences in backbone structure. In total there are 43 pairs with positive score including 16 diagonal elements.

Interestingly, the diagonal elements $m-m$ and $d-d$ substitutions that correspond to central $\alpha$-helix and central $\beta$-strands are not biased by their corresponding high frequencies owing to the normalization formula used ${ }^{36}$. Low frequencies among other PB substitutions and their good conservation, especially involving $\mathrm{N}$ and $\mathrm{C}$ caps residues of helices and strands, results in high scores in other diagonal elements.

\section{Application of the substitution matrix to identify structurally equivalent regions}

One of the most convenient ways to validate the substitution matrix is to use it for protein structural alignment and to compare the results with those obtained with other well established methods. Alignment of protein structure in terms of PBs using the substitution table and a dynamic programming approach (hereafter called PB-ALIGN) was benchmarked against the standard structural alignment methods implemented in DALI and STAMP.

When aligning two structures, PBs are assigned to the two proteins in consideration. Then, using dynamic programming approach, sequences of PBs from two proteins are 
optimally matched rather like amino acid sequence alignments. In order to quantify the extent of substitution between PBs the newly generated PB substitution matrix is used.

In order to identify a gap penalty with optimal performance a large number of $\mathrm{PB}$ alignments were generated by varying gap penalty from -1.0 to +1.0 with a step of 0.5. From manual analysis of these alignments we found out, positive penalty was highly unfavorable whereas negative penalty performed better in aligning equivalent regions. We fixed empirically the gap penalty to -0.5 , which often resulted in reasonable alignments. Analysis of the resulting alignment provides a direct way to identify structures which are equivalent or variable.

A comprehensive data set that consists of 15 protein pairs belonging to homologous families were sampled following documented test cases ${ }^{37}$ and a further 14 other protein pairs were sampled from SCOP families with a $40 \%$ sequence identity cutoff (see complete list in Methods section). Structural alignments were performed using DALI, STAMP and PB-ALIGN.

We analyzed the extent of overlapping of structurally equivalent regions by comparing (i) DALI vs STAMP (ii) DALI vs PB-ALIGN. We simply counted the number of positions within each aligned region, which were in agreement with the reference alignment from DALI.

Interestingly, PB-ALIGN was able to align as much as $75.9 \%$ of the positions that are aligned in DALI and this is comparable with the performance of STAMP which scores $77.6 \%$. Results for each of the pairwise alignments of homologous proteins from DALI and PB-ALIGN show that in more than two-third of the cases, at least $80 \%$ of the aligned positions are in common between the two methods (figure 2). First, this demonstrates that structural alignment based on the use of our PB 
substitution matrix and dynamic programming is giving reasonably comparable results when compared to standard rigid or flexible structure alignment methods. Second, this shows that PB-ALIGN can be used as a fast method to identify structural equivalences in homologous proteins.

Attempts have been made to use PB substitution scores to locate local portions in distantly related proteins that are structurally equivalent. In the first instance, we compared two distantly related alpha and beta protein $(\alpha+\beta)$ class based on SCOP classification ${ }^{38}$ from the metallohydrolase superfamily (1QH5a and 1SMLa) sharing $18 \%$ sequence identity. The lengths of these two chains are almost same (260 and 266 respectively).

An alignment of the PB sequences from these two metallohydrolases was calculated as described above. Several regions of interest in this alignment are detailed in figures 3a-c. Highlighted are four distinct PB alignment stretches. According to our PB based alignment featured at the bottom of figures $3 \mathrm{a}$ and $3 \mathrm{~b}$, these regions ( $a 1$ and $a 2$ ) would be structurally equivalent while the other two PB alignments featured in figures $3 \mathrm{a}$ and $3 \mathrm{c}$ would correspond to structurally variable regions ( $v 1$ and $v 2$ ). Superposed coordinates of 1QH5 and 1SML from STAMP are used only to highlight structural equivalent and variable regions identified by dynamic programming based alignment of PBs. Indeed, $a 1$ and $a 2$ regions circled with a solid line in figures $3 \mathrm{a}$ and $3 \mathrm{~b}$ are well superimposed. On the other hand, structures in the $v 1$ and $v 2$ regions circled with a dashed line in figure $3 \mathrm{a}$ and $3 \mathrm{c}$, as expectedly, are not well superimposed and they are not considered as structurally equivalent in the PB alignment. Simple comparison of the results of our approach with that of the SSAP method ${ }^{28}$ shows interesting results. SSAP method was successful in identifying region al but fails to identity structurally equivalent region $a 2$. Also compared to SSAP based alignment of region 
$v 2$ (not shown), the structurally variable region is more evident from PB alignment, due to poor PB scores in the region. In the case of DALI region al was well identified as equivalent but surprisingly there was no demarcation of region $v 2$ from $a 2$ as variable. In addition, the $\mathrm{C}$-terminal extension $(v l)$ adjacent to the $a l$ region displays a region that is almost equivalent between the two proteins but it appears that there are subtle conformational changes identified from the PB alignment (figure 3a). When examining this C-terminal extension in the STAMP superimposition, slight differences in backbone conformation are indeed observed (figure 3a). Once again this subtle change is not directly evident from SSAP or DALI based structure alignments.

In the second instance, two distantly related proteins (1BNKA and 1FMTB) from the all $\beta$ class FMT C-terminal domain like superfamily were studied. The lengths of these two chains are very different (200 and 108 respectively). We analyzed three different local regions from the PB alignment (figures 4a-c). Examination of the first region (figure 4a) indicates that they would be structurally equivalent regions but the 1BNK fragment is shorter than the equivalent 1FMT fragment. This is illustrated in the superimposition of the two structures. Indeed, N-cap and furthest C-cap region are well superimposed while the central helix, which is significantly longer in the 1FMT structure, is only poorly superimposed. Figure $4 \mathrm{a}$ also shows the presence of extra loop region in 1FMT as identified by the "CBE" $\mathrm{PB}$-motif in the $\mathrm{PB}$ alignment. Examination of the second region (figure 4b) from the two proteins shows that they are structurally equivalent and are indeed perfectly superimposed. Interestingly, the third region (figure 4c) is predicted as structurally equivalent by PB alignment with positive PB substitution scores. However the $\mathrm{C} \alpha$ positions are not superimposable and share no equivalent residues. Close examination of this particular region shows that 
the backbones are almost identical but poor superimposition is due to a rigid body shift (see section 3.3). This result thus indicates that despite absence of good superimposition, $\mathrm{PB}$ alignment is able, in a flexible manner, to detect structurally equivalent regions in proteins.

\section{Distinguishing conformational differences and rigid-body shifts in homologous} proteins

When two homologous structures are aligned using rigid body superposition, high $\mathrm{C} \alpha-\mathrm{C} \alpha$ deviations can result either due to conformational differences between the aligned regions or due to differences in the spatial positioning of identical conformational motifs. For example alignment of a $\alpha$-helical region and $3_{10}$ helical region in two homologues correspond to conformational difference. On the other hand, the difference in the relative position of two conserved $\alpha$-helical regions in the two homologues corresponds to rigid-body shift. Both these changes can result in high $\mathrm{C} \alpha-\mathrm{C} \alpha$ deviations.

The basic premise in distinguishing between conformational difference and rigidbody shift is that conformational differences are characterized by high root mean square deviations (RMSD) of pentapeptide regions and poor PB substitution scores. Difference in spatial orientation of structurally conserved segments is characterized by high $\mathrm{C} \alpha-\mathrm{C} \alpha$ deviations in the pentapeptides, but, good (favoured) PB substitution scores. Thus simple transformation of superimposed structures into PB alignment provides a novel and direct way to rapidly detect these two situations.

Detailed description of how PBs can be used to address this issue is documented in the supplementary material of this manuscript. Three examples are provided; (i) relative orientation of $\mathrm{C}$-terminal lobe with respect to $\mathrm{N}$-terminal lobe in 
endothiapepsins (E.C. 3.4.23.6) ; (ii) rigid body shifts and conformational changes in two distantly related proteins of class II aminoacyl tRNA synthetase $\mathrm{N}$ domain ; (iii) structural alterations between active and inactive states of cyclic AMP dependent protein kinase.

\section{Discussion}

Arriving at a meaningful measurement of the probabilities for short structural motifs to change conformation in topologically equivalent regions is only possible if local backbone of a set of structurally aligned proteins is decoyed in terms of a structural alphabet. This issue is, to our knowledge, addressed for the first time here using protein blocks ${ }^{20}$. Interestingly, because of the methodology used to construct the matrix, direct evaluation of equivalences between homologous structures is possible which is not the case for the HMM based matrix derived from SA-Search ${ }^{32}$. The derived substitution matrix here suggests that perceivable conformational changes occur even in topologically equivalent regions of homologous proteins. This is indicated by negative scores of most of the off diagonal elements (table 2) despite considering topologically equivalent regions in rigid body superimposition from PALI database.

Because protein blocks allow encoding of protein 3D structures into 1D sequences, these can, interestingly, be manipulated rather like amino acids sequences. This approach has been explored here, namely for structure comparison and alignment. For PB alignment to be relevant, the availability of a biologically meaningful PB substitution matrix was a prerequisite. This was ensured by the methodology used for its construction. Even though, the matrix required further validation in terms of its performance to align pairs of protein structures in comparison to well-established 
methods. Aligning protein structures by aligning PB sequences using dynamic programming is different from aligning secondary structural elements because PBs describe more precisely the backbone conformation in coil regions and $\mathrm{N}$ or $\mathrm{C}$ caps of regular helices or strands ${ }^{18}$. Hence it is expected to be more efficient than a $3 \times 3$ matrix. Alignment, using PBs, is achieved here in a flexible manner and performs comparably to other robust flexible structural alignment methods like in DALI ${ }^{29}$ or SSAP $^{28}$. However, it is noteworthy that the actual implementation of PB alignment is expected to fail in detecting domain swapping situations. The originality in our approach resides in the methodology used, which, besides being very intuitive, is very different from those implemented in $\mathrm{DALI}^{29}$ or $\mathrm{SSAP}^{28}$.

Importantly, these two methods are being routinely used for structure comparison on a large-scale basis via web services. Concurrently, application of PB substitution matrix in protein fold recognition is expected to be a useful venture. This has recently been tested on a large-scale basis. We showed that the efficiency rate to mine similar fold proteins from SCOP using 1D representation as sequence of PBs varies from $86.1 \%$ to 93.6\% (Tyagi et al., submitted) thus further validating our approach and substitution matrix. A web service that implements this approach (Tyagi et al., submitted) is available at http://bioinformatics.univ-reunion.fr/PBE/.

\section{Conclusions}

In this paper, we demonstrated, using a structural alphabet, the usefulness of encoding 3D structure into 1D space through the use of a substitution matrix.

Such a substitution table is shown to be useful in distinguishing conformational changes and rigid-body shifts of structural motifs in homologous proteins. Its 
application is also demonstrated in terms of characterization of structural differences involving rigid body movements between active and inactive forms of an enzyme.

Using 1D representation of 3D structure combined with our substitution matrix and simple dynamic programming, we were, even in the case of two homologues with very different sequence lengths, able to locate regions of structural similarity, highlight subtle change in conformations within aligned regions and to identify regions of no structural similarity. Though robust methods such as DALI ${ }^{29}$ and SSAP 28 are quite sensitive and effective in rapid detection of common folds and structural motifs, the applications presented in this paper clearly highlights the original and informative nature of the derived 16x16 substitution matrix and gives good indication of its strength in protein structure analysis.

This work has important implications in comparative modeling of loops. Besides it can used to add meaning to non-superimposed (variable) regions, in databases of structurally aligned proteins like PALI, by finding structural equivalence in these regions.

As an extension of our work, we are working towards arriving at gross flexible global and local structural alignment of distantly related proteins with optimized gap penalties. Similarly, general consideration on the rationale for constructing such a matrix is presently being addressed likewise amino acid substitution matrices ${ }^{39}$ namely in the field of PBs compositional bias. We are also currently investigating the distribution properties of raw $\mathrm{PB}$ alignment scores against randomized datasets in both local and global alignment schemes in order to arrive at a genuine statistical measurement of alignment significance. 


\section{Electronic supplementary material}

The complete list of pairs of protein domains that was used in this study are given in supplementary material 1 .

Detailed description of how PBs can be used to distinguish between conformational differences and rigid body shifs is documented in supplementary material 2 of this manuscript. Three examples are provided; (i) relative orientation of C-terminal lobe with respect to $\mathrm{N}$-terminal lobe in endothiapepsins (E.C. 3.4.23.6) ; (ii) rigid body shifts and conformational changes in two distantly related proteins of class II aminoacyl tRNA synthetase $\mathrm{N}$ domain ; (iii) structural alterations between active and inactive states of cyclic AMP dependent protein kinase. Five new figures are provided here.

In addition, supplementary material 3 is provided as a zip file containing stereo images of figure 1, superimposed PDB coordinates from STAMP for figures 3 and 4, as well as for the supplementary figures $6,7,8$ and 9 together with the PB alignments and corresponding Rasmol scripts.

\section{Acknowledgements}

We thank Mr. C. Sairam Swamy for providing the code for dynamic programming and Rajesh Thangudu for his suggestions on the work. NS is an International Senior Fellow of the Wellcome Trust, London. He thanks the authorities of Reunion University for the visiting professorship in the laboratory of BO. Manoj TYAGI is supported by a PhD grant from the Conseil Régional de La Réunion. We thank anonymous reviewers for fruitful suggestions. 


\section{References}

1. Jones TA, Thirup S. Using known substructures in protein model building and crystallography. Embo J 1986;5(4):819-822.

2. Levitt M. Accurate modeling of protein conformation by automatic segment matching. J Mol Biol 1992;226(2):507-533.

3. Unger R, Sussman JL. The importance of short structural motifs in protein structure analysis. J Comput Aided Mol Des 1993;7(4):457-472.

4. Han KF, Baker D. Recurring local sequence motifs in proteins. J Mol Biol 1995;251(1):176-187.

5. Unger R, Harel D, Wherland S, Sussman JL. A 3D building blocks approach to analyzing and predicting structure of proteins. Proteins 1989;5(4):355-373.

6. Bystroff C, Baker D. Prediction of local structure in proteins using a library of sequence-structure motifs. J Mol Biol 1998;281(3):565-577.

7. Karchin R, Cline M, Mandel-Gutfreund Y, Karplus K. Hidden Markov models that use predicted local structure for fold recognition: alphabets of backbone geometry. Proteins 2003;51(4):504-514.

8. Hunter CG, Subramaniam S. Protein local structure prediction from sequence. Proteins 2003;50(4):572-579.

9. Etchebest C, Benros C, Hazout S, de Brevern AG. A structural alphabet for local protein structures: improved prediction methods. Proteins 2005;59(4):810-827.

10. Camproux AC, Gautier R, Tuffery P. A hidden Markov model derived structural alphabet for proteins. J Mol Biol 2004;339(3):591-605.

11. Kolodny R, Koehl P, Guibas L, Levitt M. Small libraries of protein fragments model native protein structures accurately. J Mol Biol 2002;323(2):297-307.

12. Tuffery P, Guyon F, Derreumaux P. Improved greedy algorithm for protein structure reconstruction. J Comput Chem 2005;26(5):506-513. 
13. Park BH, Levitt M. The complexity and accuracy of discrete state models of protein structure. J Mol Biol 1995;249(2):493-507.

14. Fourrier L, Benros C, de Brevern AG. Use of a structural alphabet for analysis of short loops connecting repetitive structures. BMC Bioinformatics 2004;5(1):58.

15. Camproux AC, Brevern AG, Hazout S, Tuffery P. Exploring the use of a structural alphabet for structural prediction of protein loops. Theor Chem Acc 2001;106(1-2):28-35.

16. Camproux AC, Tuffery P, Buffat L, Andre C, Boisvieux JF, Hazout S. Analyzing patterns between regular secondary structures using short structural building blocks defined by a hidden Markov model. Theor Chem Acc 1999;101(1-2):33-40.

17. de Brevern AG, Hazout S. 'Hybrid protein model' for optimally defining 3D protein structure fragments. Bioinformatics 2003;19(3):345-353.

18. de Brevern AG, Valadie H, Hazout S, Etchebest C. Extension of a local backbone description using a structural alphabet: a new approach to the sequence-structure relationship. Protein Sci 2002;11(12):2871-2886.

19. de Brevern AG, Hazout S. Compacting local protein folds with a "hybrid protein model". Theor Chem Acc 2001;106(1-2):36-47.

20. de Brevern AG, Etchebest C, Hazout S. Bayesian probabilistic approach for predicting backbone structures in terms of protein blocks. Proteins 2000;41(3):271-287.

21. de Brevern AG. New assessment of a structural alphabet. In Silico Biology 2005;5:26.

22. Karchin R. Evaluating local structure alphabets for protein structure prediction. PhD Computer Science 2003.

23. de Brevern AG, Wong H, Tournamille C, Colin Y, Le Van Kim C, Etchebest C. A structural model of a seven-transmembrane helix receptor: the Duffy 
antigen/receptor for chemokine (DARC). Biochim Biophys Acta 2005;1724(3):288-306.

24. Schuchhardt J, Schneider G, Reichelt J, Schomburg D, Wrede P. Local structural motifs of protein backbones are classified by self-organizing neural networks. Protein Eng 1996;9(10):833-842.

25. Rost B. Prediction in 1D: secondary structure, membrane helices, and accessibility. Methods Biochem Anal 2003;44:559-587.

26. Lo Conte L, Smith TF. Visible volume: a robust measure for protein structure characterization. J Mol Biol 1997;273(1):338-348.

27. Taylor WR, Orengo CA. A holistic approach to protein structure alignment. Protein Eng 1989;2(7):505-519.

28. Taylor WR, Orengo CA. Protein structure alignment. J Mol Biol 1989;208(1):1-22.

29. Holm L, Sander C. Protein structure comparison by alignment of distance matrices. J Mol Biol 1993;233(1):123-138.

30. Orengo CA, Taylor WR. A local alignment method for protein structure motifs. J Mol Biol 1993;233(3):488-497.

31. Ye J, Janardan R, Liu S. Pairwise protein structure alignment based on an orientation-independent backbone representation. J Bioinform Comput Biol 2004;2(4):699-717.

32. Guyon F, Camproux AC, Hochez J, Tuffery P. SA-Search: a web tool for protein structure mining based on a Structural Alphabet. Nucleic Acids Res 2004;32(Web Server issue):W545-548.

33. Balaji S, Sujatha S, Kumar SS, Srinivasan N. PALI-a database of Phylogeny and ALIgnment of homologous protein structures. Nucleic Acids Res 2001;29(1):61-65. 
34. Gowri VS, Pandit SB, Karthik PS, Srinivasan N, Balaji S. Integration of related sequences with protein three-dimensional structural families in an updated version of PALI database. Nucleic Acids Res 2003;31(1):486-488.

35. Russell RB, Barton GJ. Structural features can be unconserved in proteins with similar folds. An analysis of side-chain to side-chain contacts secondary structure and accessibility. J Mol Biol 1994;244(3):332-350.

36. Johnson MS, Overington JP. A structural basis for sequence comparisons. An evaluation of scoring methodologies. J Mol Biol 1993;233(4):716-738.

37. Shindyalov IN, Bourne PE. Protein structure alignment by incremental combinatorial extension (CE) of the optimal path. Protein Eng 1998;11(9):739-747.

38. Murzin AG, Brenner SE, Hubbard T, Chothia C. SCOP: a structural classification of proteins database for the investigation of sequences and structures. J Mol Biol 1995;247(4):536-540.

39. Altschul SF, Wootton JC, Gertz EM, Agarwala R, Morgulis A, Schaffer AA, Yu YK. Protein database searches using compositionally adjusted substitution matrices. Febs J 2005;272(20):5101-5109. 


\section{Tables}

Table 1. Ideal values of $\phi$ and $\Psi$ dihedral angles (in degrees) that characterize the 16 Protein Blocks as described by de Brevern et al., (2000).

\begin{tabular}{ccccccccc}
\hline & \multicolumn{7}{c}{ dihedral angles } \\
\cline { 2 - 9 } Protein Block & $\psi_{\mathrm{n}-2}$ & $\phi_{\mathrm{n}-1}$ & $\psi_{\mathrm{n}-1}$ & \multicolumn{1}{c}{$\phi_{\mathrm{n}}$} & \multicolumn{1}{c}{$\psi_{\mathrm{n}}$} & $\phi_{\mathrm{n}+1}$ & $\psi_{\mathrm{n}+1}$ & $\phi_{\mathrm{n}+2}$ \\
\hline$(a)$ & 41.14 & 75.53 & 13.92 & -99.80 & 131.88 & -96.27 & 122.08 & -99.68 \\
$(b)$ & 108.24 & -90.12 & 119.54 & -92.21 & -18.06 & -128.93 & 147.04 & -99.90 \\
$(c)$ & -11.61 & -105.66 & 94.81 & -106.09 & 133.56 & -106.93 & 135.97 & -100.63 \\
$(d)$ & 141.98 & -112.79 & 132.2 & -114.79 & 140.11 & -111.05 & 139.54 & -103.16 \\
$(e)$ & 133.25 & -112.37 & 137.64 & -108.13 & 133.00 & -87.30 & 120.54 & 77.40 \\
$(f)$ & 116.4 & -105.53 & 129.32 & -96.68 & 140.72 & -74.19 & -26.65 & -94.51 \\
$(g)$ & 0.40 & -81.83 & 4.91 & -100.59 & 85.50 & -71.65 & 130.78 & 84.98 \\
$(h)$ & 119.14 & -102.58 & 130.83 & -67.91 & 121.55 & 76.25 & -2.95 & -90.88 \\
$(i)$ & 130.68 & -56.92 & 119.26 & 77.85 & 10.42 & -99.43 & 141.4 & -98.01 \\
$(j)$ & 114.32 & -121.47 & 118.14 & 82.88 & -150.05 & -83.81 & 23.35 & -85.82 \\
$(k)$ & 117.16 & -95.41 & 140.40 & -59.35 & -29.23 & -72.39 & -25.08 & -76.16 \\
$(l)$ & 139.20 & -55.96 & -32.7 & -68.51 & -26.09 & -74.44 & -22.60 & -71.74 \\
$(m)$ & -39.62 & -64.73 & -39.52 & -65.54 & -38.88 & -66.89 & -37.76 & -70.19 \\
$(n)$ & -35.34 & -65.03 & -38.12 & -66.34 & -29.51 & -89.10 & -2.91 & 77.90 \\
$(o)$ & -45.29 & -67.44 & -27.72 & -87.27 & 5.13 & 77.49 & 30.71 & -93.23 \\
$(p)$ & -27.09 & -86.14 & 0.30 & 59.85 & 21.51 & -96.30 & 132.30 & -92.91 \\
\hline
\end{tabular}


Table 2. Normalized substitution frequencies expressed as log-odds scores between any two protein blocks as determined by structure-based pairwise alignments of homologous proteins of known three-dimensional structure from PALI database.

\begin{tabular}{|c|c|c|c|c|c|c|c|c|c|c|c|c|c|c|c|c|}
\hline $\begin{array}{c}\text { Protein } \\
\text { blocks }\end{array}$ & $a$ & $b$ & $c$ & $d$ & $e$ & $f$ & $g$ & $h$ & $i$ & $j$ & $k$ & $l$ & $m$ & $n$ & $O$ & $p$ \\
\hline$a$ & 2.28 & & & & & & & & & & & & & & & \\
\hline$b$ & -0.12 & 2.49 & & & & & & & & & & & & & & \\
\hline$c$ & 0.54 & -0.21 & 1.69 & & & & & & & & & & & & & \\
\hline$d$ & -0.29 & -0.44 & 0.17 & 1.35 & & & & & & & & & & & & \\
\hline$e$ & -1.59 & -0.48 & -1.10 & -0.36 & 3.05 & & & & & & & & & & & \\
\hline$f$ & -0.54 & -1.53 & -0.39 & -0.49 & 0.75 & 2.21 & & & & & & & & & & \\
\hline$g$ & 0.31 & -0.73 & 0.18 & -1.29 & 1.37 & -0.33 & 3.25 & & & & & & & & & \\
\hline$h$ & -1.14 & 0.20 & -1.63 & -1.20 & 0.66 & -0.34 & -0.74 & 3.07 & & & & & & & & \\
\hline$i$ & 0.39 & 0.24 & -1.11 & -1.12 & -1.15 & -1.07 & -0.19 & -0.92 & 3.37 & & & & & & & \\
\hline$j$ & -1.15 & 0.32 & -1.03 & -0.92 & -0.76 & -0.34 & -0.51 & 1.18 & 1.54 & 3.74 & & & & & & \\
\hline$k$ & -1.75 & -0.03 & -2.45 & -2.63 & -0.38 & -0.04 & -1.39 & 0.51 & -0.15 & 0.07 & 2.52 & & & & & \\
\hline$l$ & -0.60 & 0.04 & -2.21 & -1.56 & -1.76 & -0.33 & -0.74 & -0.36 & -0.22 & -0.12 & 0.19 & 2.24 & & & & \\
\hline$m$ & -2.40 & -2.98 & -2.70 & -5.20 & -4.75 & -2.14 & -1.10 & -2.93 & -3.15 & -2.00 & -1.02 & -0.68 & 1.06 & & & \\
\hline$n$ & -1.40 & -0.83 & -1.68 & -3.07 & -0.58 & -1.99 & 1.07 & -1.07 & -0.97 & -0.44 & -0.56 & -0.27 & -0.77 & 3.65 & & \\
\hline$o$ & -0.54 & -0.55 & -0.65 & -2.66 & -2.48 & -1.41 & -0.01 & 0.96 & -0.89 & -0.48 & -1.71 & 0.06 & -1.26 & 0.26 & 3.36 & \\
\hline$p$ & -0.36 & 0.33 & -0.01 & -2.10 & -2.22 & -1.91 & 0.47 & -1.81 & 1.32 & 0.60 & -1.35 & -1.23 & -1.10 & 0.36 & 0.24 & 2.83 \\
\hline
\end{tabular}




\section{Figures}

Figure 1 - Backbone comparison of PBs.

(a) Superimposed backbone structures of PB $a$ (black) and $g$ (grey), where matrix gives positive score for substitution between $a$ to $g$. (b) Backbone structure of PB $e$ (black) and $f$ (grey) having positive substitution score. (c) Backbone structure of $\mathrm{PB} a$ (black) and $j$ (grey) having negative substitution score.

\section{Figure 2 - Comparison of PB-ALIGN, STAMP and DALI.}

Comparison of structural alignment against DALI using STAMP (y-axis) and PBALIGN (x-axis). Each axis represents the percentage of aligned positions that are in agreement with alignment from DALI.

\section{Figure 3 - Identification of structurally equivalent regions between 1QH5 and} 1SML

Superposed structures of $1 \mathrm{QH} 5$ and $1 \mathrm{SML}$ are used here to highlight structurally equivalent and variable regions identified by PB-based alignment. (a) The regions from the two proteins circled with a solid line are identified as equivalent according to the alignment using PBs shown at the bottom of the figure. The $\mathrm{C}$ terminal extension of this region is shown in another circled region (dashed line). (b)The region $a 2$ of the two proteins that is also identified, using PBs, as equivalent are shown in black for 1QH5a (region 101-119) and in grey for 1SMLa (region 150-168). (c) Identification of variable regions by PB alignment for 1QH5 (region 82-90 in grey) and 1SML (region 109-139 shown in black). STAMP superposition indeed shows that these two regions are structurally non-equivalent. Files are provided for this figure (see additional material for details). 
Figure 4 - Identification of structurally equivalent regions between 1FMT and 1BNK

Superposed structures of $1 \mathrm{FMT}$ and $1 \mathrm{BNK}$ are used here to highlight structural equivalent and conformationally variable regions identified by dynamic programming based alignment of PBs. (a) Aligned helical region from 1FMTb (218-236 region shown in black) and 1BNKa (region 93-105 shown in grey) indicating longer helical and loop region in 1FMTb as identified by PB alignment. Circled region with dashed line shows the presence of extra loop in C termini of 1FMTb (region 231-233) which corresponds to the extra "CBE" PB-motif in the alignment. (b) The highlighted region from the two proteins is identified as equivalent from PB alignment and is shown in black for protein 1FMTb (region 245-253) and in grey for protein 1BNKa (region 119-127). (c) Similar structural regions of 1FMTb (region 281-295 shown in black) and 1BNKa (region 169-181 shown in grey) but which are not superimposable by rigid body superposition. Regions discussed above uses original residue numbering as given in PDB coordinate files. Files are provided for this figure (see additional material for details). 